\title{
Climate policy without intertemporal dictatorship: Chichilnisky Criterion vs. Classical Utilitarianism in DICE
}

\author{
W. J. Wouter Botzen \\ Institute for Environmental Studies \\ VU University De Boelelaan 1087 \\ 1081 HV Amsterdam \\ The Netherlands \\ wouter.botzen@vu.nl \\ $\&$ \\ Utrecht University School of Economics \\ Utrecht University, The Netherlands \\ and \\ Jeroen C.J.M. van den Bergh \\ ICREA, Barcelona, Spain \\ $\&$ \\ Institute of Environmental Science and Technology \\ Universitat Autònoma de Barcelona, Spain \\ $\&$ \\ Faculty of Economics and Business Administration \\ $\&$ the Institute for Environmental Studies \\ VU University Amsterdam, The Netherlands \\ jeroen.bergh@uab.es \\ and \\ Graciela Chichilnisky \\ Columbia Consortium for Risk Management \\ Columbia University, New York NY 10037 USA \\ $\&$ \\ SIEPR and Department of Economics, Stanford University \\ chichilnisky1@gmail.com
}

October 2017

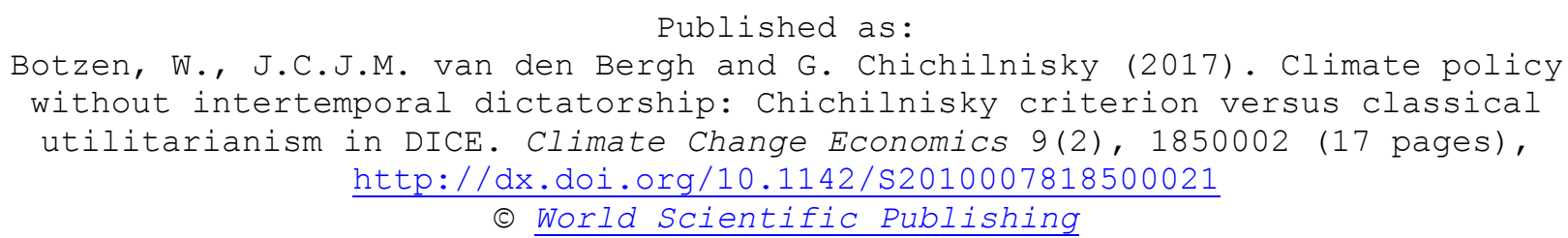




\title{
Climate policy without intertemporal dictatorship: Chichilnisky Criterion vs. Classical Utilitarianism in DICE
}

\begin{abstract}
Unlike discounting and the damage function, the social welfare function has not received so much attention in the debate on climate economics. An important challenge has been to combine efficiency and equity considerations in a single social welfare framework. The Chichilnisky criterion is one way to resolve this. We consider its implementation in the climate-economy model DICE, and compare results for different damage functions, namely the standard one in DICE and the one proposed by Weitzman implying potential large climate damages at high temperature increases. We calculate optimal climate policy for different parameter settings and compare the results with those under the green golden rule (only final utility matters) and classical utilitarianism (no discounting). Optimal emission abatement trajectories turn out to be very different between standard discounted utilitarianism, classical utilitarianism, and Chichilnisky specifications. The results are very sensitive to the damage function, the climate sensitivity parameter, and especially the "Chichilnisky weight" given to utility of generations in the far future. We discuss conditions and reasons for preferring either classical utilitarianism or the Chichilnisky criterion, and conclude that a critical factor is the time horizon used in climate policy analysis. Adopting sustainable preferences as formalised by the Chichilnisky criterion in climate policy analysis has the advantage that the very long term implications of greenhouse gases in the atmosphere on the environment and human welfare are not downplayed.
\end{abstract}

\section{Highlights:}

- Optimal climate policy is derived using the Chichilnisky social welfare criterion.

- Emission abatement depends on the weight of utility in the far future.

- Climate policy according to Chichilnisky can be more stringent than classical utilitarianism.

- The green golden rule advices immediate drastic cuts in emissions.

Key words: Chichilnisky welfare criterion, classical utilitarianism, climate change, DICE model, Weitzman damage function. 


\section{Introduction}

Unlike discounting and the economic damage function, the social welfare function has received rather sparse attention in the debate on climate economics. This is surprising, as very different choices of the welfare function are possible, which give more or less weight to risk aversion to uncertain climate change impacts, or to concerns about the trade-off between efficiency, equity and sustainability (Tol, 2002). The latter trade-off has received very much attention in the literature on sustainable development (Toman et al., 1995). In the context of climate change, arguably the most worrisome sustainability problem currently faced by humanity, various alternative social welfare functions have been tested. Botzen and van den Bergh (2014) give an account of these studies and show that a more systematic approach is needed to deal with at least 14 core approaches that span the space of possible social welfare functions. Here we focus on the treatment of intergeneration equity and long-term sustainability, which is especially relevant for climate policy because of the very long term nature of impacts caused by greenhouse gas emissions (Roemer, 2011; Dietz and Asheim, 2012).

The formal method followed takes a discounted utility or net present value (NPV) approach as the starting point and modifies it by adding a component and a set of weights to it. This is motivated by the recognition in sustainability studies that we need to combine efficiency and equity considerations in a single social welfare framework. The Chichilnisky $(1996,1997)$ criterion represents a concrete method to achieve this. It maximizes a weighted average of a discounted sum of utilities plus the terminal utility value. This criterion is motivated by axioms stating that present decisions should not disregard the far future, while sensitivity to present welfare implications should be accounted for. For a theoretical analysis of axioms of the this criterion we refer to the original work of Chichilnisky $(1996,1997)$. The objective of our study is to examine how the implementation of the Chichilnisky criterion influences advice about optimal climate policy and how this depends on important parameter assumptions. This requires examining how particular climate dynamics and feedback (utility damage) will work out in terms of the Chichilnisky criterion compared to other criteria. Since climate dynamics is complex, there is no other way than working with concrete climate-economy models. We consider its implementation in the climate-economy model DICE (Nordhaus, 2008) as it is fairly simple, and has been shown to be suitable for addressing theory-motivated questions. It allows to study how changes in, or additions to, the DICE framework alter its basic findings and policy advice. It also allows for 
comparison of results under distinct damage functions, such as of the "Nordhaus" and more extreme "Weitzman" type. We will further examine sensitivity of the Chichilnisky criterion to the adopted discount rate, in view of this having received much debate.

The DICE (Dynamic Integrated Climate-Economy) model by William Nordhaus is one of the most studied economic models of optimal climate policy (Nordhaus, 1991, 1994, 2008; Nordhaus and Boyer, 2000). DICE has been much debated while alternative formulations have been proposed and analyzed.

We calculate optimal climate policy for different parameter settings, focusing on the damage function and the weight given to utility of a generation in the far future. We further compare the results with those obtained for a social welfare function that gives equal weight to each generation, i.e. classical utilitarianism or no intergenerational discounting. As no information is provided in articles by Chichilnisky on which weights to use, we undertake sensitivity analysis on this, including considering only the final utility term as the social welfare function, which comes down to giving the full weight to this term, also known as the "green golden rule".

The remainder of this paper is organized as follows. Section 2 briefly introduces the DICE model. Section 3 presents the Chichilnisky criterion and summarizes the (mainly) theoretical literature on it. Section 4 performs simulations with the Chichilnisky-adapted DICE model, and compares its behaviour with NPV and classical utilitarianism approaches. Section 5 concludes.

\section{Climate economics with DICE}

In the early 1990s William Nordhaus developed DICE, an integrated assessment model (IAM) combining a Ramsey-type optimal economic growth model and an aggregate climate module. This describes the cause-effect chain from economic production, through carbon dioxide $\left(\mathrm{CO}_{2}\right)$ emissions and atmospheric concentrations, to temperature changes, sea level rise, and (extreme) weather patterns and events, and ultimately economic damages. The model reflects damages in various economic sectors, notably agriculture, farming, forestry, tourism, water, energy and real estate (human settlements), as well as impacts on human health and ecosystems. DICE allows calculating optimal time paths of emission reduction and carbon taxes. DICE has seen many adaptations and extensions, dealing with learning, irreversible investments, endogenous technological change, adaptation, and alternative damage functions (e.g., Pizer, 1999; Popp, 2005; de Bruin et al., 2009; Botzen and van den Bergh, 2012; Hwang et al., 2013; Ackerman et al., 2013). 
We apply the DICE-2008 model (Nordhaus, 2008) in the optimization software GAMS. We follow the certainty-equivalent approach of Nordhaus (2008), which uses expected values of all parameters. In this way, we can directly compare our findings with the original DICE results. Unless stated otherwise we use the standard parameters of DICE (Nordhaus, 2008).

As in most standard IAMs, the social welfare function in DICE is grounded in discounted utilitarianism. It can be formalized as:

$W=\sum_{t=1}^{T \max } U[c(t), L(t)] R(t)$

The utility function is of the form $U[c(t), L(t)]=L(t)\left[c(t)^{1-\gamma} /(1-\gamma)\right]$ where $c(t)$ and $L(t)$ are per capita consumption and population at time $t$, respectively, while $\gamma$ is the elasticity of marginal utility of consumption which in all of our runs equals the standard value in DICE of 2 (Nordhaus, 2008). Population growth is exogenous in DICE. The social time preference discount factor is $R(t)=(1+\rho)^{-t}$, where $\rho$ is the pure rate of social time preference. The appropriate value of $\rho$ has been hotly debated, especially since Stern (2007) showed that using a lower value $(0.1 \%)$ than was common (like $1.5 \%$ in DICE-2008) on grounds of intergenerational equity implies much larger optimal reductions in greenhouse gas emissions. In addition to applying (1) with the standard DICE discount rate which we will call "standard NPV”, we apply classical utilitarianism in DICE by setting $\rho=0 .^{1}$

Another important component of the DICE model is the damage function. The standard damage function used by Nordhaus (2008) has the following specification:

$\Omega(t)=1 /\left[1+0.0028 T_{A T}(t)^{2}\right]$

Here $\Omega(t)$ represents one minus the fraction of aggregate output (in trillion US\$) lost due to climate change, $t$ is time (decades in DICE 2008), and $T_{A T}(t)$ is the global mean surface temperature above

\footnotetext{
${ }^{1}$ This means zero discounting over the finite time horizon of DICE, with zero weight given to later generations. This can be regarded to approximate, in a theoretical sense, an approach that discounts the entire infinite temporal series of $U$ with a small positive discount rate (Posner, 2005).
} 
pre-industrial levels. Based on expert advice, Weitzman (2012) proposes an alternative damage function which we will use as well in our simulations, namely:

$\Omega(t)=1 /\left[1+\left(T_{A T}(t) / 20.46\right)^{2}+\left(T_{A T}(t) / 6.081\right)^{6.754}\right]$

This effectively introduces a tipping point where the damages function describes large impacts beyond $6{ }^{\circ} \mathrm{C}$ temperature increase. Here the specification generates approximately $50 \%$ damage at $6^{\circ} \mathrm{C}$. Figure 1 illustrates the different behaviours of the functions in (2) and (3). Although hardly visible, the Weitzman damage is slightly lower than Nordhaus damage for temperature increases from 0.5 up to a little above $2.5^{\circ} \mathrm{C}$, while Weitzman damages are larger for higher increases in temperature.

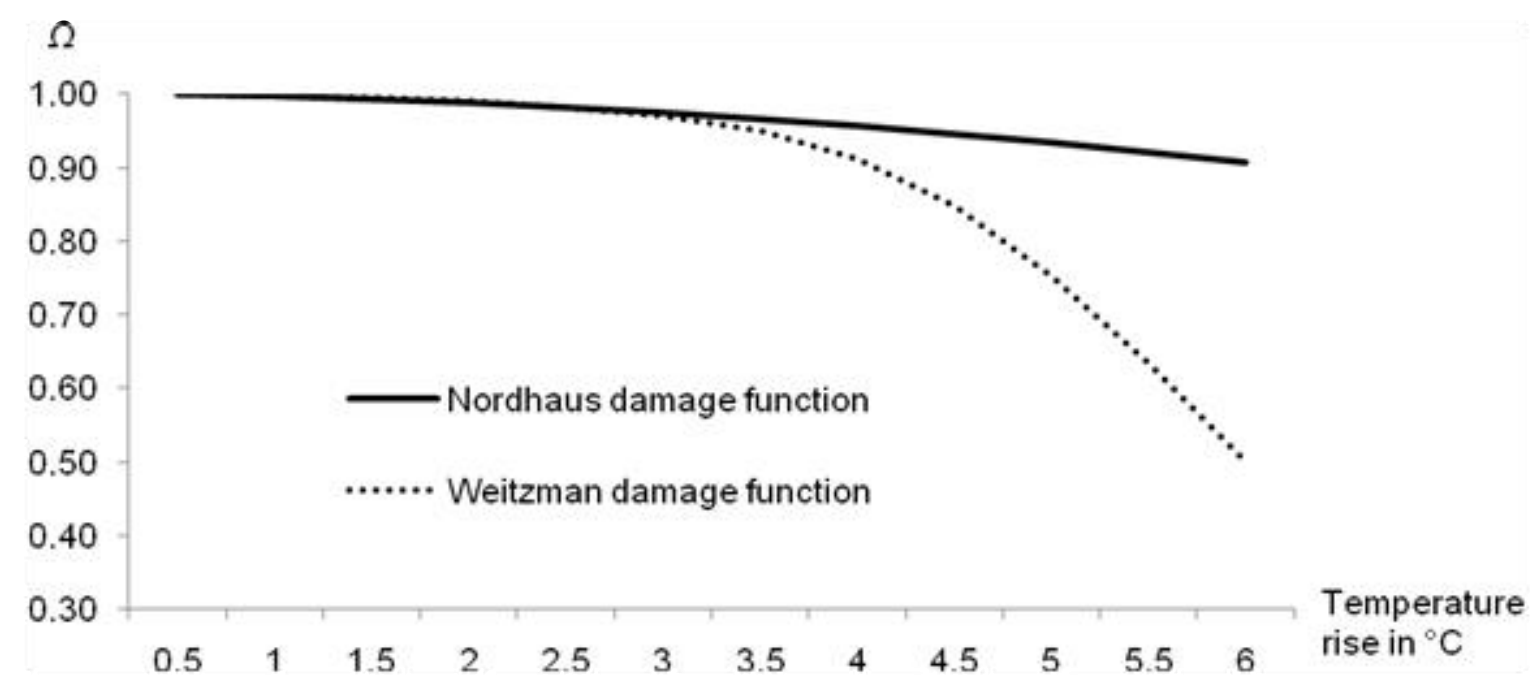

Figure 1. Comparison of behaviour of the Nordhaus and Weitzman damage functions

Note: The lower $\Omega$, the higher climate damage.

\section{The Chichilnisky welfare criterion}

Chichilnisky $(1996,1997)$ proposed axioms to assure a sustainable development. These required that neither the present nor future generations dictate outcomes, in other words an equal treatment of the present and the future. Based on this, she derives an alternative social welfare specification which includes the discounted utility framework as an extreme case: it maximizes a weighted average of a discounted sum of utilities plus the terminal utility value (assuming a finite time horizon). This welfare criterion $W$ can be expressed as (Chichilnisky, 1996): 
$W(c)=\alpha \sum_{t=1}^{\infty} \rho^{t-1} u_{t}\left(c_{t}\right)+(1-\alpha) \lim _{t \rightarrow \infty} u_{t}\left(c_{t}\right)$

with weight $\alpha$ for which $0<\alpha<1$ holds. In order to examine sensitivity of results to the discount rate value, we apply criterion (4) using the standard DICE discount rate and compare this with standard DICE results under (1) as well as with results when the lower Stern (2007) discount rate is applied.

This criterion is clearly sensitive to what happens in both the present and the (distant) future. It thus overcomes the shortcoming of discounted utilitarianism (the NPV criterion) in which the present dictates the outcome in disregard for the future due to assigning a monotonously decreasing weight to utility over time. The underlying welfare axioms are defined by Chichilnisky as sustainable preferences. In models with finite time horizons the Chichilnisky criterion coincides with discounted utilitarianism, i.e. a net present value based on discounting future streams of instantaneous utility. The terminal utility term is known as the "green golden rule" (equivalent to $\alpha=0)$, represented as max $\lim _{t \rightarrow \infty} u\left(C_{t}\right)$ (Chichilnisky et al., 1995). In this study we examine the implications of the Chichilnisky criterion for the abatement of greenhouse gasses. ${ }^{2}$ In the numerical exercise later on in the paper we use a pragmatic approach, namely replacing the limitto-infinity part of the final term of the Chichilnisky criterion by a non-discounted utility term at the final horizon of the problem studied, which is the year 2200 which is the last period in the 2008 version of the DICEmodel that we use (Nordhaus, 2008). At that time a long run economic steady state has been reached in the DICE model which is based on a standard Solow growth model with a Cobb-Douglas production function and exogenous technological change (Estrada et al., 2015). ${ }^{3}$ We tested for so-called boundary effects of choosing this last time period, which did not appear to be affecting our results. ${ }^{4}$ Evidently, the theoretical limit of utility in the infinite future cannot be captured by a numerical model. Our approximation provides, thought, relevant insights by

\footnotetext{
${ }^{2}$ Saving rates are the same in all of our model runs since we use the standard DICE model parameters in which saving rates are calibrated to meet observed savings (Nordhaus, 2008).

${ }^{3}$ One can view our application as assuming that in the year 2200 a stationary steady state economy is reached, as has been predicted to occur in the future by prominent economic thinkers, like Adam Smith, John Stuart Mill, and John Maynard Keynes.

${ }^{4}$ The sensitivity of the results to time-boundary (edge) effects associated with the finite time horizon was examined by approximating the terminal utility term by the utility in period 59 , which did not alter the results. Note further that a global optimum solution was found for the model optimization in GAMS for all model runs reported in this paper.
} 
illustrating how attaching different utility weights to the very far future influences optimal climate policy.

Our approach, and arguably any other numerical approach, to the Chichilnisky criterion cannot deal with infinite time. We feel the infinite case is not so relevant anyway as climaterelevant decisions by humans will always be motivated by finite time horizons, even if long. It may be seen as an advantage that both the Chichilnisky and classical utilitarianism approaches in our paper deal with finite time horizons, as it allows for comparisons since similarly restrictive assumptions are made with regard to the time horizon. That is, it would be worse if one approach adopted an infinite and the other a finite time perspective.

The literature on the Chichilnisky criterion is predominantly theoretical (e.g., Le Kama et al., 2014). An exception is Tol (1999) who implements an adjusted version of the Chichilnisky criterion in FUND by defining welfare in the last period as the square of the deviation of $\mathrm{CO}_{2}$ concentrations in the year 2200 with a safe value of 550. Weight $\alpha$ is then set such that a businessas-usual concentration of 1793ppm takes away half of the present value of welfare of the first generations (the NPV term in equation 4), while for later generations the weight falls linearly to zero for the 21sth and last generation (Tol, 1999). However, this approach resembles more a pure precautionary principle, as Tol himself also explains (see also Tóth, 2000).

An unresolved difficult issue is how to set the weight $(1-\alpha)$ assigned to the long-run future (utility). Chichilnisky $(2009$, p.5) suggests that it can be seen as “... the marginal utility of the resource at the point of extinction. This is the point where the resource is presumably most valuable." For the context of climate change this suggests the point where the climate system becomes very unstable or where the biosphere and human system become severely damaged, for example, suggesting a point where temperature increases with 6 degrees and beyond (Weitzman, 2012). What would be the numerical value of the marginal utility of a resource close to extinction? Obviously, this question is very difficult to answer. Clearly the value would be very high which would justify a value of $(1-\alpha)$ close to 1 , which implies that $\alpha$ is extremely small. Nevertheless the interpretation of $\alpha$ does not result in an unambiguous numerical value for $\alpha$, which is why we conduct an extensive sensitivity analysis on this parameter that includes extreme values. 


\section{Simulations with the adapted DICE model}

Table 1 shows the temperature rise and associated economic output of the "optimal policy" run of the DICE model for three welfare approaches, namely the original Nordhaus welfare function, classic utilitarianism, and the specification proposed by Chichilnisky. A comparison of the standard model run and classical utilitarianism shows the sensitivity of the results to the adopted discount rate: in particular, temperature is stabilized at an increase of about $3.5^{\circ} \mathrm{C}$ in the standard discounted utilitarian welfare specification, while this is approximately $1{ }^{\circ} \mathrm{C}$ lower if no discounting is applied (i.e. $\rho=0$ ).

Optimal climate policy with the Chichilnisky criterion can be more or less stringent than classical utilitarianism depending on the weight attached to the terminal utility value. Large values of $\alpha$ imply results similar to those of the standard run. Perhaps it makes intuitive sense to give the same weight to the "present" and far future. We operationalize this by giving an equal weight to the terminal utility value as to the other time periods - i.e. $(1-\alpha)=1 / 60$ or alternatively $\alpha=59 / 60-$ which results in identical results (not shown in Table 1) as standard NPV. In other words, the results shown at standard NPV in Table 1 also apply to Chichilnisky with $(1-\alpha)=1 / 60 .^{5}$

It should be realized that while $(1-\alpha)=1 / 60$ implies an equal weight of the sum of the discounted utility term and the undiscounted terminal utility term, the values of these utility terms may be quite different. The reason is that whereas the first term is influenced by discounting, the second term is not. Moreover, the total consumption level increases over time, due to economic and population growth, positively affecting values of utility in later generations. In fact, a much larger population being exposed to climate impacts in the far future could be a reason for adopting lower values of $\alpha$, even though it gives little guidance on the exact weight to be used. More importantly, extremely low values of $\alpha$ would be justified for making (1- $\alpha$ ) represent the marginal utility value of a resource near the point of extinction. Small changes in $\alpha$ may have large changes in DICE model results, because of the high value of final utility which would increase over time due to population and economic growth.

\footnotetext{
${ }^{5}$ An alternative specification could be to normalize the weight of the discounted utility term in equation 4 to equal 1. This implies that the relative weight of the sum of the discounted utility term is high, resulting in very similar findings to the standard NPV results reported in Table 1, even when the Chichilnisky term has a high weight of $\alpha=0.1^{11}$; in particular, the emission control rate is only 0.01 higher than standard NPV from the year 2105 onwards. Since this alternative specification basically delivers the same results as the standard DICE social welfare function and because our specification of the weight $\alpha$ in equation 4 is closer to the theoretical Chichilnisky criterion, we prefer the specification of weights in equation 4.
} 
Climate policy is more stringent than standard NPV for small $\alpha$ weights, as these imply giving more importance to the terminal utility term. For example, $\alpha=0.1^{9}$ results in a slightly lower temperature increase after the year 2155. $\alpha=0.1^{10}$ results in an optimal temperature rise that is close to classical utilitarianism. An even smaller weight $\alpha=0.1^{11}$ results in a maximum optimal temperature rise of about $1{ }^{\circ} \mathrm{C}$ which is considerably lower than classical utilitarianism. Climate policy is very stringent early on as the emission control rate of 1 in 2015 indicates. Results with this small weight are the same as the green golden rule, which is not surprising given that $\alpha$ is very close to 0 . It is clear from Table 1 that the more stringent climate policies obtained under Chichilnisky with $\alpha<0.1^{9}$ prevent large temperature increases that go at the expense of long-term economic output.

Of course, such small values of $\alpha$ raise questions about why it is so close to zero. One should note, however, that "small" and "close to zero" are relative concepts. They depend on the specification of utility, climate dynamics, and feedback (damage) in DICE, as well as on the number of generations as captured by the first term. Moreover, we do not claim that the $\alpha$ should be so small, but simply mention threshold values above for which the optimal strategy alters.

Table 1. Results of the "optimal policy" run of the DICE model with the standard damage function and various social welfare specifications and parameter values

\begin{tabular}{|l|rrrrr|}
\hline & 2015 & 2055 & 2105 & 2155 & 2195 \\
\hline Standard NPV: & & & & & \\
\hline Temperature rise in ${ }^{\circ} \mathrm{C}$ & 0.95 & 1.78 & 2.69 & 3.30 & 3.47 \\
Gross output in trillion US\$ & 70 & 138 & 270 & 494 & 781 \\
Emission control rate & 0.16 & 0.27 & 0.44 & 0.68 & 0.93 \\
Classical utilitarianism: & & & & & \\
\hline Temperature rise in ${ }^{\circ} \mathrm{C}$ & 0.95 & 1.69 & 2.40 & 2.58 & 2.36 \\
Gross output in trillion US\$ & 69 & 141 & 277 & 507 & 815 \\
Emission control rate & 0.25 & 0.41 & 0.67 & 1 & 1 \\
Chichilnisky with $\alpha=0.1^{19}$ : & & & & & \\
\hline Temperature rise in ${ }^{\circ} \mathrm{C}$ & 0.95 & 1.80 & 2.71 & 3.30 & 3.27 \\
Gross output in trillion US\$ & 69 & 141 & 278 & 506 & 799 \\
Emission control rate & 0.18 & 0.27 & 0.46 & 0.71 & 1 \\
Chichilnisky with $\alpha=0.1^{10}$ : & & & & & \\
\hline Temperature rise in ${ }^{\circ} \mathrm{C}$ & 0.96 & 1.76 & 2.75 & 2.55 & 2.31 \\
Gross output in trillion US\$ & 70 & 141 & 271 & 266 & 318 \\
Emission control rate & 0.05 & 0.29 & 1 & 1 & 1
\end{tabular}




\begin{tabular}{|l|rrrrr|} 
Chichilnisky with $\alpha=0.1^{11}$ : & & & & & \\
Temperature rise in ${ }^{\circ} \mathrm{C}$ & 0.90 & 1.04 & 1.04 & 1.00 & 0.94 \\
Gross output in trillion US $\$$ & 56 & 92 & 150 & 231 & 322 \\
Emission control rate & 1 & 1 & 1 & 1 & 1 \\
Chichilnisky green golden rule: & & & & & \\
\hline Temperature rise in ${ }^{\circ} \mathrm{C}$ & 0.90 & 1.04 & 1.04 & 1.00 & 0.94 \\
Gross output in trillion US $\$$ & 64 & 92 & 213 & 231 & 322 \\
Emission control rate & 1 & 1 & 1 & 1 & 1 \\
\hline
\end{tabular}

We conduct a sensitivity analysis by estimating optimal climate policy for the same welfare specifications and parameter values using the Weitzman damage function in equation (3) instead of the Nordhaus DICE one in (2). The results of this exercise are shown in Table 2. Optimal temperature rises in the NPV run are lower than for the standard damage function. This can be expected since the high temperature increases of more than $3^{\circ} \mathrm{C}$, which are optimal under the standard damage function, are avoided when the Weitzman function is used that implies large damages for such high temperature rises (Figure 1). The differences between the temperature rise in the NPV and classical utilitarianism runs are smaller than when the standard damage function is used, suggesting that sensitivity of the results to the discount rate is less in case the damage function allows for more extreme climate change impacts. The Chichilnisky results are more sensitive now to the weight $\alpha$ than is the case for the standard damage function. Table 2 shows that optimal temperature rises with $\alpha=0.1^{9}$ are slightly above those in the NPV run. This finding suggests that giving some more weight to the far future compared with the NPV run does not necessarily imply more drastic emission cuts with this damage function. This result could be due to a terminal time effect where we seek to increase consumption in the last period by decreasing the emission control rate in intermediate periods, reflected by a drop in the emission control rate at that time. Reducing the weight to $\alpha=0.1^{10}$ results in a lower maximum temperature rise than under the NPV and classical utilitarianism, suggesting that in that case the aim of reducing longterm climate damages dominates. Temperature rise is even lower when $\alpha=0.1^{11}$ which produces results that are not the same as the green golden rule, while these results were identical with the standard damage function.

Applying Chichilnisky with $\alpha=0.1^{11}$ results in slightly higher optimal temperatures with the Weitzman damage function compared with the standard damage function. This may appear to be a surprising result. However, it should be kept in mind that the Weitzman damage function 
results in lower climate damages than the standard function for temperature rises below $2.5^{\circ} \mathrm{C}$, which is the relevant range for temperature rises under both damage functions when $\alpha=0.1^{11}$. The Weitzman damage function allows for some higher optimal temperatures in this case, but sill limits temperature rise to $2.2^{\circ} \mathrm{C}$. Applying the green golden rule results in the same optimal temperatures regardless of the damage function, and implies drastic emission cuts under both damage functions applied here.

Table 2. Results of the "optimal policy" run of the DICE model with the Weitzman damage function and various social welfare specifications and parameter values

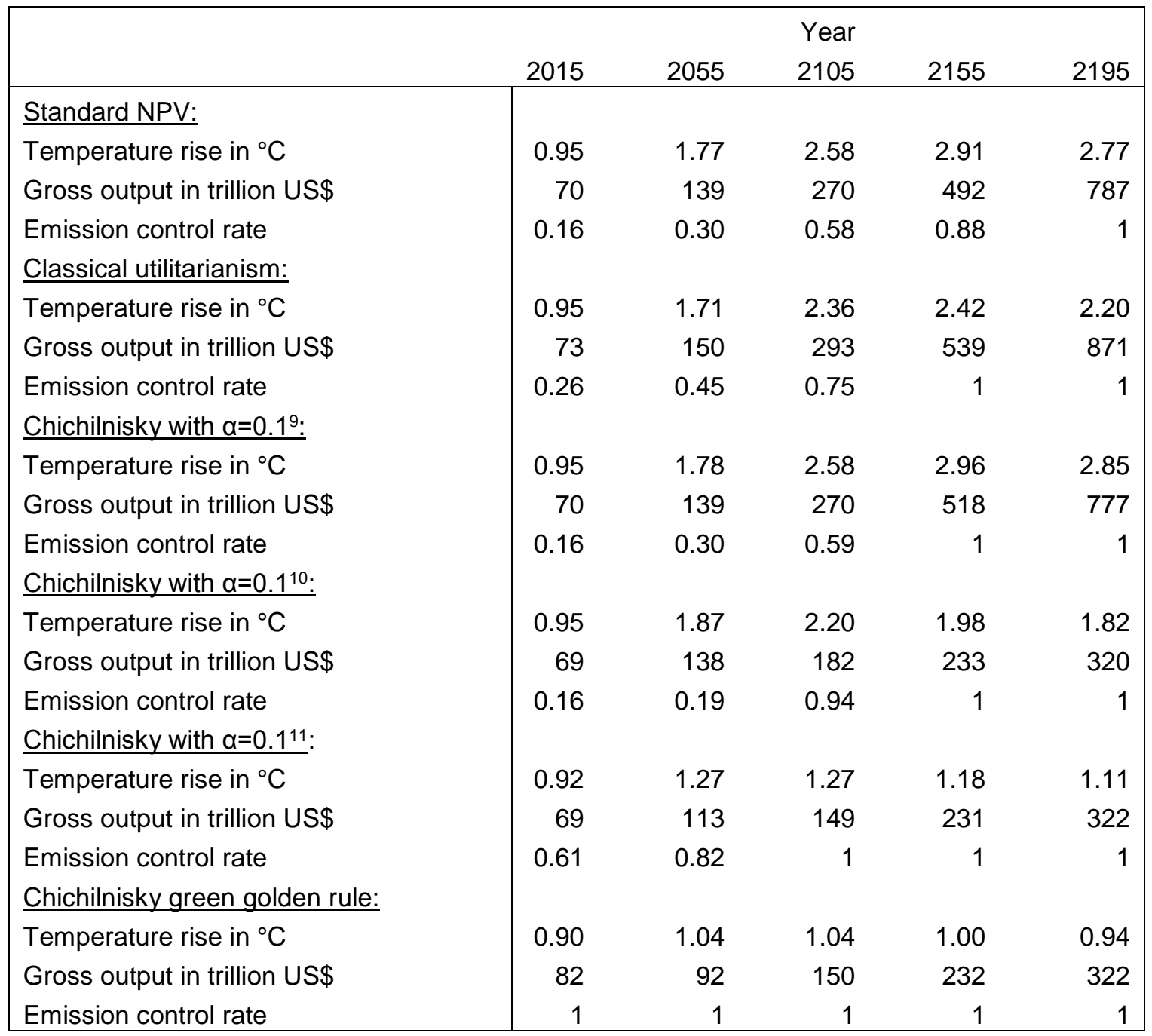

Another main uncertainty is the climate sensitivity parameter which indicates the long term warming that results from a doubling of $\mathrm{CO}_{2}$ emissions. This parameter is set equal to $3^{\circ} \mathrm{C}$ in the standard DICE model. Table 3 shows the results of increasing climate sensitivity to $4^{\circ} \mathrm{C}$ which 
according to the IPCC (2014) falls within a likely range. The higher climate sensitivity implies that temperature increase is always higher than with the standard climate sensitivity results even for the most stringent climate policy under the Chichilnisky criterion. Moreover, under all welfare criteria early emission control rates are much more stringent if climate sensitivity is higher, reflecting higher benefits of emission abatement. Sometimes the Chichilnisky criterion results in a more lenient climate policy in later periods to boost consumption in the last period. Nevertheless, optimal maximum temperature rise according to the Chichilnisky criterion is always lower than under standard NPV.

Table 3. Results of the "optimal policy" run of the DICE model with the standard damage function and various social welfare specifications, for parameter values assuming a higher climate sensitivity

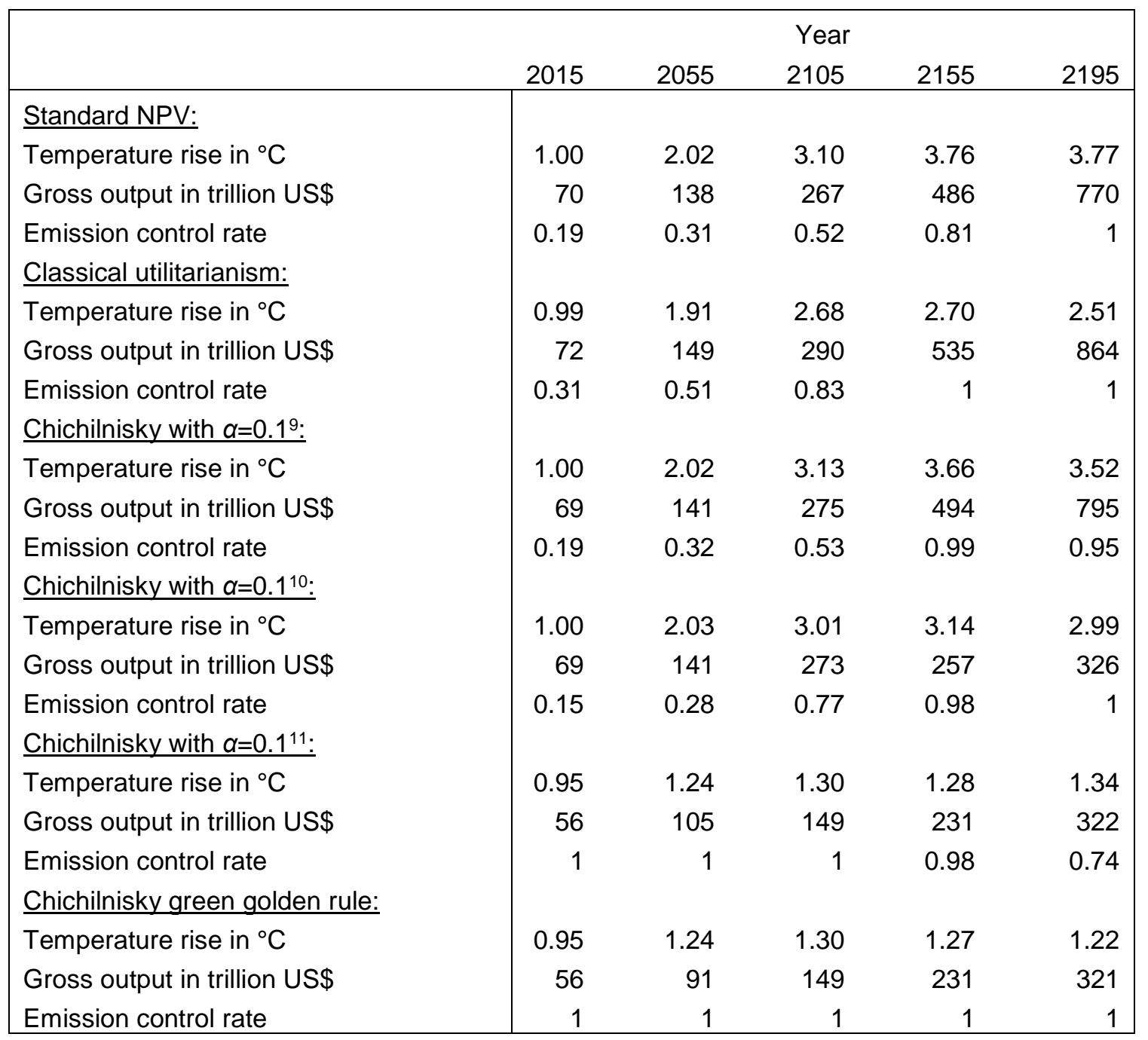


Next, we examine sensitivity of using the Chichilnisky criterion with respect to the adopted discount rate. These model runs use again the standard DICE damage function, but the utility discount rate parameter $\rho$ is reduced from the standard DICE value of $1.5 \%$ to $0.1 \%$. This lower value is consistent with the discount rate used by Stern (2007), consistent with Botzen and van den Bergh (2012). This reduction of the discount rate implies a smaller optimal temperature rise with $\alpha=0.1^{9}$ compared with the standard DICE discount rate. The same applies to using weight $\alpha=0.1^{10}$ which results in a maximum temperature increase of about $2.8^{\circ} \mathrm{C}$ using standard DICE discounting and $2.6^{\circ} \mathrm{C}$ using Stern discounting. Interestingly an opposite effect on climate policy of lowering the discount rate is found for Chichilnisky weight $\alpha=0.1^{11}$. Using the standard discount rate the terminal utility value completely dominates the sum of discounted utility component of the social welfare function resulting in a climate policy that is as stringent as $\alpha=0$ (Table 1). However, this domination of the final term does not happen with the Stern discount rate resulting in a higher temperature rise (Table 4) than using the standard discount rate (Table 1). The reason is that lowering the discount rate implies that the sum of discounted utility component of the Chichilnisky social welfare function increases in relative importance to the terminal utility value (equation 4). Therefore, the usual result in standard discounted utilitarianism that a lower discount rate implies a more stringent climate policy does not always hold for the Chichilnisky welfare criterion and depends on the weight $\alpha$. Evidently, results of $\alpha=0$ are not sensitive to the discount rate used since in that case the non-discounted terminal utility value receives the full weight regardless.

Table 4. Results of the "optimal policy" run of the DICE model with the standard damage function and various social welfare specifications and parameter values, using Stern discounting

\begin{tabular}{|l|rrrrr|}
\hline & \multicolumn{5}{c|}{ Year } \\
\hline Chichilnisky with $\alpha=0.1^{19}$ : & 2015 & 2055 & 2105 & 2155 & 2195 \\
\hline Temperature rise in ${ }^{\circ} \mathrm{C}$ & 0.95 & 1.70 & 2.42 & 2.65 & 2.42 \\
Gross output in trillion US\$ & 69 & 141 & 277 & 506 & 814 \\
Emission control rate & 0.24 & 0.40 & 0.65 & 1 & 1 \\
Chichilnisky with $\alpha=0.1^{10}$ : & & & & & \\
Temperature rise in ${ }^{\circ} \mathrm{C}$ & 0.94 & 1.66 & 2.39 & 2.57 & 2.32 \\
Gross output in trillion US\$ & 69 & 141 & 277 & 506 & 814 \\
Emission control rate & 0.34 & 0.40 & 0.66 & 1 & 1 \\
Chichilnisky with $\alpha=0.1^{11}:$ & & & & &
\end{tabular}




\begin{tabular}{|l|rrrrr|} 
Temperature rise in ${ }^{\circ} \mathrm{C}$ & 0.90 & 1.87 & 2.00 & 1.79 & 1.66 \\
Gross output in trillion US\$ & 70 & 140 & 160 & 242 & 332 \\
Emission control rate & 1 & 1 & 1 & 1 & 1 \\
\hline
\end{tabular}

Figure 2 shows the optimal $\mathrm{CO}_{2}$ emissions for different social welfare specifications in DICE using the standard damage function and utility discount rate. Applying classical utilitarianism results in a smooth emissions curve that has a shape similar to that under standard NPV, but is always below the latter. Emission pathways under Chichilnisky are very sensitive to the weight attached to the terminal utility value. The emissions curve of $\alpha=0.1^{9}$ lies slightly above the standard NPV curve until the year 2105, and afterwards emissions decline more steeply. Under Chichilnisky with weight $\alpha=0.1^{10}$ emissions are allowed to rise more steeply over the course of this century, but drop strongly around 2100. This suggests that the final utility term dominates the decision criterion from the year 2100 onwards since the emission pathway becomes the same as under the green golden rule. Increasing the weight of the final utility even more (Chichilnisky with weight $\alpha=0.1^{11}$ ) results in an optimal emission pathway that is already after the year 2025 exactly the same as the green golden rule (not shown in Figure 2 because it would hamper readability of the figure). The green golden rule implies immediate drastic cuts of greenhouse gas emissions. 


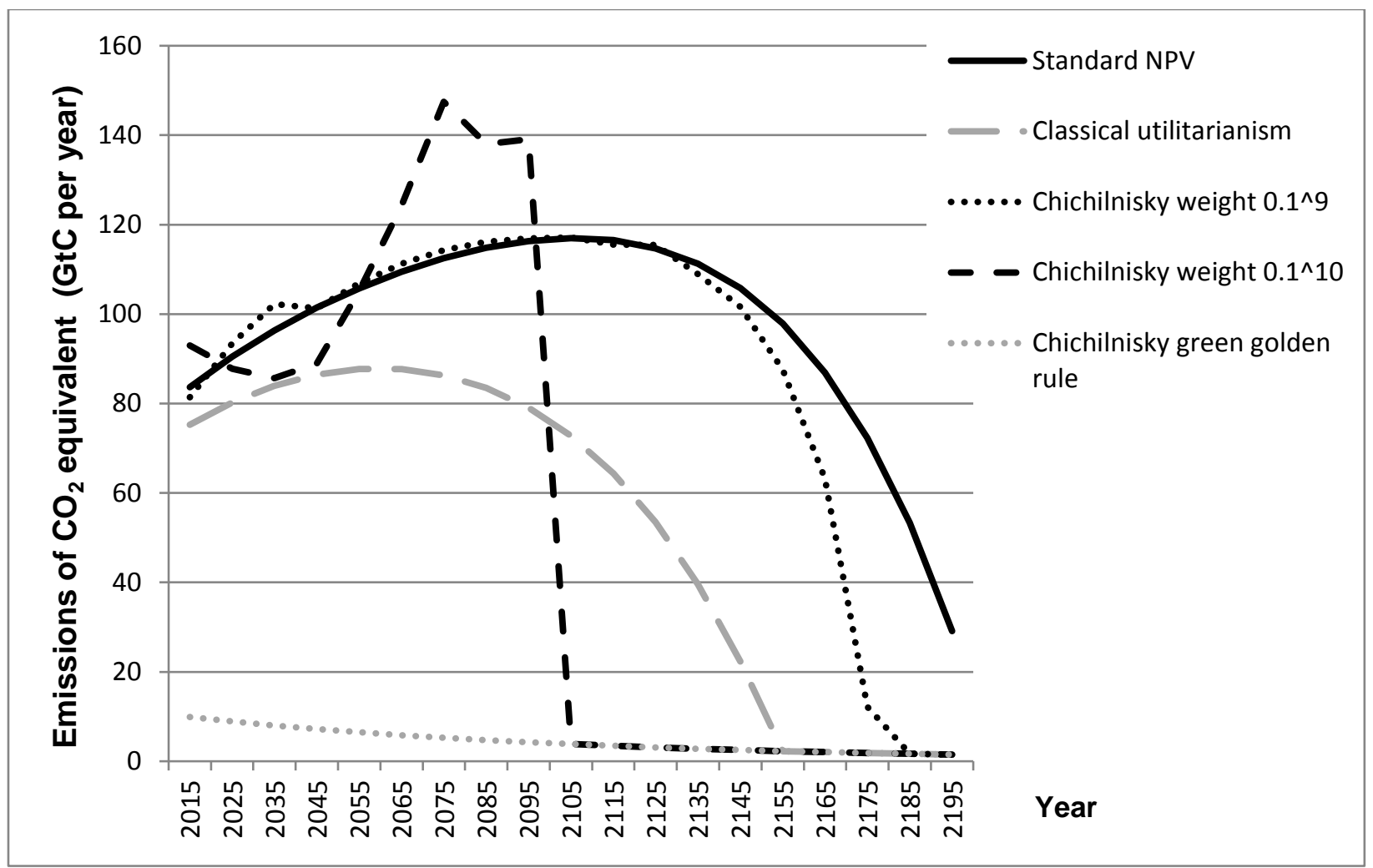

Figure 2. Optimal $\mathrm{CO}_{2}$ emissions in DICE with the standard damage model for different social welfare specifications and parameter values

Note: The curve with Chichilnisky weight $0.1^{11}$ coincides with the Chichilnisky green golden rule and is, therefore, not shown separately.

The comparison of the curves for the Chichilnisky weight $0.1^{10}$ and classical utilitarianism is insightful. It shows that the latter, because it gives more weight to intermediate time periods relative to early time periods, stimulates an optimal emissions pattern that is lower already initially, to reduce damages for intermediate time periods (or generations). The Chichilnisky criterion in this case leads to a more extreme strategy, namely emitting a lot initially to benefit early generations who have a high utility weight in the NPV criterion, and then at some time radically shifting to very low emissions (consistent with the green golden rule) to benefit the terminal utility (or generation). In a way, the combination of NPV and terminal utility term in the Chichilnisky criterion mean for this specific weight value that early and final time periods (generations) dominate the outcomes, where intermediate ones receive less weight and therefore less concern. The distribution of emissions is less extreme for classical utilitarianism as all generations receive an equal weight. This can be a reason to prefer the classical utilitarianism over the Chichilnisky 
term, also because the motivation of the weight in the latter is difficult, and we have shown that the policy results are very sensitive to its specific value.

Existing studies which are most comparable with our approach are Tol (1999), Tol (2013), and Dietz and Asheim (2012). Tol (1999) applies a modification of the Chichilnisky criterion in the climate-economy model FUND (see Section 3). His results show that a Chichilnisky-like criterion results in a more stringent climate policy than the standard NPV and than model runs with lower than standard discount rates. We find similar results for the Chichilnisky criterion compared with standard NPV for low-values of weight $\alpha$. We additionally show that using a lower than standard discount rate may result in more or less stringent climate policies dependent on weight $\alpha$. However, Tol's implementation of the Chichilnisky criterion as a target greenhouse gas concentration level may be interpreted as an application of the precautionary principle (Tóth, 2000) which deviates from our approach.

Tol (2013) applies a Bentham-Rawls criterion proposed by Alvarez-Cuadrado and Van Long (2009), which is a social welfare function that maximizes the weighted sum of net present value of welfare and the welfare of the worst-off generation. This may be interpreted as a special case of the Chichilnisky criterion in case the last generation is the poorest. This could only happen if climate change impacts were to offset growth, something which is unlikely according to Tol (2013). Hence, the Bentham-Rawls criterion will generally generate outcomes that differ from those of our approach. A numerical illustration by Tol (2013) indeed shows that the BenthamRawls criterion only results in more stringent emission abatement than standard discounted utilitarianism when extreme parameters are used, notably, a high probability of decreasing utility over time, a high weight on the utility of the worst-off generation, and very high climate change damage.

Dietz and Asheim (2012) adapt the Nordhaus (2008) DICE model to implement a sustainable discounted utilitarianism criterion. This welfare function only applies a zero discount rate as in classical utilitarianism if the present is better off than the future, and a positive discount rate if the future is better off than the present. This latter discount rate condition is consistent with the rank- rank-discounted utilitarian criterion proposed by Zuber and Asheim (2012), who also proposed the alternative of using negative discount rates when future generations are worse off than the present. Dietz and Asheim (2012) combined the implementation of the sustainable discounted utilitarianism criterion with an extensive sensitivity analysis of uncertain parameters in 
DICE using Monte Carlo simulations. This risk analysis implies that the probability that a generation is better off than its descendants is non-negligible. Using the standard DICE discount rate, their results show that sustainable discounted utilitarianism results in slightly more stringent emission reductions than under standard discounted utilitarianism. For example, in the year 2150 the optimal emission control rates are about 0.65 and 0.7 under sustainable and standard discounted utilitarianism, respectively (Dietz and Asheim, 2012). We find that the Chichilnisky criterion can result in much more stringent climate policies with early emission control rates equal or close to 1 , dependent on weight $\alpha$.

\section{Conclusions}

Replacing in standard DICE the NPV by the Chichilnisky criterion does not alter the optimal climate policy if weights attached to the terminal utility value are small. Using only the final term of the Chichilnisky criterion, i.e. giving weight 1 to this and 0 to the discounted utility part, leads to a much more stringent policy, the so-called green golden rule. Using classical utilitarianism (zero discounting over the entire analysis period) leads to a less stringent policy than this, but to a more stringent policy than the NPV criterion. Climate policy under Chichilnisky can be more or less stringent than classical utilitarianism depending on the weight given to the far future.

Overall, our study of the Chichilnisky criterion in DICE and extensive sensitivity analysis of other model parameters contribute to a broader literature showing that a variety of particular modifications in the DICE model can generate rapid emission control as the optimal strategy. A review by van den Bergh and Botzen (2014) illustrates that stringent climate policy are generally an economically efficient outcome in IAMs when low discount rates are used for intergenerational equity, and high climate damages are accounted for through appropriate damage function or climate sensitivity specifications. Moreover, once DICE generates the $100 \%$ emission control strategy as optimal due to changes in one of these discussed assumptions, it becomes insensitive to further modifications. That is, with multiple modifications, the strong policy response is overdetermined, and additional modifications are not additive, i.e. do not alter the optimal strategy.

The pragmatic application of the Chichilnisky criterion here did not change results versus those of the NPV criterion for non-extreme weights. The strongest effects (differences with NPV) were found for a very high weight for the final Chichilnisky term. Sensitivity of results to the weight are larger when the Weitzman damage function is applied that allows for more extreme 
climate change impacts. If standard DICE is too optimistic about the damage function, climate sensitivity or growth of emissions without policy, extreme climate change with very high temperature changes is likely to create considerable differences in policy suggestions between NPV and Chichilnisky welfare criteria. This is particularly true if a Weitzman damage function is used.

A very high weight for the final term in the Chichilnisky criterion, as used by us to generate certain results, may be criticized as unrealistic. However, this can be nuanced in several ways. First, the further in time the final term is, the more the weight needs to compensate for the accumulation of (discounted) utilities at earlier points in time. In other words, the weight logically increases when environmental policies are evaluated that have very long run effects, like climate change. But for a higher discount factor, this compensation evidently would be smaller. That is, the weight decreases with the discount factor. Second, as was argued in Section 3, in the vein of Chichilnisky's suggestion that the weight represents “... the marginal utility of the resource at the point of extinction.", the value is indeed high if one considers very extreme climate change endangering basic life support functions of humans as well as for other species - witness the very high rates of biodiversity loss predicted under scenarios of extreme climate change (e.g., Stern, 2007).

We learn three things from this exercise. First, in terms of policy implications, for large weights $\alpha$ the Chichilnisky criterion effectively reduces to the NPV criterion. Second, the outcomes for the Chichilnisky criterion are highly sensitive in the range of very small weights. Third, in the latter case, the early and final time periods (generations) dominate the outcomes, while intermediate ones receive less weight and therefore less concern. Moreover, varying the discount factor (applying the Stern discount rate) we find that the usual result in standard discounted utilitarianism that a lower discount rate implies a more stringent climate policy does not always hold for the Chichilnisky welfare criterion and depends on the weight $\alpha$. The distribution of emissions is more uniform or less extreme under classical utilitarianism as here all generations receive an equal weight. Therefore, one may prefer classical utilitarianism over the Chichilnisky approach when a smooth emission reduction path is desired, for instance, because it reduces economic shocks and associated costs for society. Classical utilitarianism moreover has the advantage of avoiding two subjective choices, namely that of discount rate and Chichilnisky weight. On the other hand, various arguments have been put forward for positive discounting, at 
least in the short term. The advantage of the Chichilnisky approach is that it combines this with explicit concern for long-term sustainability. Adopting sustainable preferences as formalised in the Chichilnisky criterion in climate policy analysis has the advantage of explicitly giving attention to, and thus not downplaying, the very long term implications of greenhouse gases in the atmosphere on the environment and human welfare.

\section{References}

Ackerman, F., Stanton, E.A., Bueno, R. (2013). Epstein-Zin utility in DICE: Is risk aversion irrelevant to climate policy? Environment and Resource Economics, 56: 73-84.

Alvarez-Cuadrado, F., Van Long, N. (2009). A mixed Bentham-Rawls criterion for intergenerational equity: theory and implications. Journal of Environmental Economics and Management, 58(2): 154-168.

Botzen, W.J.W., van den Bergh, J.C.J.M. (2012). How sensitive is Nordhaus to Weitzman? Climate policy in DICE with an alternative damage function. Economics Letters, 117: 372374.

Botzen, W.J.W., van den Bergh, J.C.J.M. (2014). Specifications of social welfare in economic studies of climate policy: Overview of criteria and evaluation of policy insights. Environmental and Resource Economics, 58(1): 1-33.

Chichilnisky, G. (1996). An axiomatic approach to sustainable development. Social Choice and Welfare, 13(2): 231-257.

Chichilnisky, G. (1997). What is sustainable development. Land Economics, 73(4): 467-491.

Chichilnisky, G. (2009). Avoiding Extinction: Equal treatment of the present and the future.

Economics: The Open-Access, Open-Assessment E-Journal, 3, 2009-32, http://www.economics-ejournal.org/economics/journalarticles/2009-32.

de Bruin, K.C., R.B. Dellink, Tol, R.S.J. (2009). AD-DICE: an implementation of adaptation in the DICE model. Climatic Change, 95: 63-81.

Dietz, S., Asheim, G.B. (2012). Climate policy under sustainable discounted utilitarianism. Journal of Environmental Econonomics and Managagement, 63: 321-335. 
Estrada, F., Tol, R.S.J., Gay-García, C. (2015). The persistence of shocks in GDP and the estimation of the potential economic costs of climate change. Environmental Modelling \& Software, 69: 155-165.

Hwang, C., Reynès, F., Tol, R.S.J. (2013). Climate policy under fat-tailed risk: An application of DICE. Environment and Resource Economics, 56: 415-436.

IPCC (2014). Climate Change 2014: Synthesis Report. Fifth Assessment Report of the Intergovernmental Panel on Climate Change. Cambridge University Press, New York.

Le Kama, A.A., Ha-Huy, T., Le Van, C., Schubert, K. (2014). A never-decisive and anonymous criterion for optimal growth models. Economic Theory, 55: 281-306.

Nordhaus, W.D. (1991). To slow or not to slow: the economics of the greenhouse effect. The Economic Journal, 101(407): 920-937.

Nordhaus, W.D. (1994). Managing the Global Commons: The Economics of Climate Change. The MIT Press, Cambridge, MA.

Nordhaus, W.D. (2008). A Question of Balance: Weighing the Options on Global Warming Policies. Yale University Press, New Haven.

Nordhaus, W.D., Boyer, J. (2000). Warming the World: Economic Models of Global Warming. The MIT Press, Cambridge, MA.

Pizer, W.A. (1999). The optimal choice of climate change policy in the presence of uncertainty. Resource and Energy Economics, 21: 255-287.

Popp, D. (2005). ENTICE: endogenous technological change in the DICE model of global warming. Journal of Environmental Economics and Management, 48: 742-768.

Posner, R. (2005). Catastrophe: Risk and Response. Oxford University Press, Oxford.

Roemer, J.E. (2011). The ethics of intertemporal distribution in a warming planet. Environment and Resource Economics, 48: 363-390. 
Stern, N. (2007). The Economics of Climate Change: The Stern Review. Cambridge University Press, Cambridge.

Toman, M.A., Pezzey, J., Krautkraemer, J.(1995). Neoclassical economic growth theory and "sustainability". In: D.W. Bromley (ed.), Handbook of Environmental Economics. Blackwell, Oxford.

Tol, R.S.J. (1999). Time discounting and optimal control of climate change: An application of FUND. Climatic Change, 41: 351-362.

Tol, R.S.J. (2002). Welfare specifications and optimal control of climate change: An application of fund. Energy Economics, 24: 367-376.

Tol, R.S.J. (2013). Climate policy with Bentham-Rawls preferences. Economics Letters, 118: 424428.

Tóth, F.L. (2000). Intergenerational equity and discounting. Integrated Assessment, 1: 127-139.

Weitzman, M. (2012). GHG targets as insurance against catastrophic climate damages. Journal of Public Economic Theory, 14(2): 221-244.

Zuber, S., Asheim, G.B. (2012). Justifying social discounting: The rank-discounted utilitarian approach. Journal of Economic Theory, 147(4): 1572-1601. 